\title{
Rho-Related GTP-Binding Protein RhoB
}

National Cancer Institute

\section{Source}

National Cancer Institute. Rho-Related GTP-Binding Protein RhoB. NCI Thesaurus. Code C17331.

Rho-related GT P-binding protein RhoB (196 aa, $\sim 22 \mathrm{kDa}$ ) is encoded by the human RHOB gene. This protein plays a role in the modulation of protein trafficking and the inhibition of tumorigenesis. 